\title{
Voz hipernasal y regurgitación nasal aguda: Reporte de un caso clínico y revisión de la literatura
}

\author{
Hypernasal speech and acute nasal regurgitation: \\ A case report and a review of the literature
}

Natalie Thone $\mathbf{M}^{1}$, Pablo Cabello E , María Santander B², Jorge Astudillo S', Matías Winter D'.

\section{RESUMEN}

La voz hipernasal y la regurgitación nasal son síntomas de disfunción velofaríngea. Ésta puede tener múltiples causas: anatómicas, neurológicas o funcionales. Se describe el caso de una paciente de sexo femenino, de 13 años, que se presenta con voz hipernasal y regurgitación nasal aguda. Al examen físico se evidencia inmovilidad del velo del paladar derecho sin otros hallazgos neurológicos. El estudio con resonancia nuclear magnética de cerebro y punción lumbar fueron normales. Se diagnosticó una incompetencia velofaríngea aguda transitoria, de probable etiología viral. La paciente evolucionó de forma favorable con mejoría clínica progresiva. La incompetencia velofaríngea a causa de una paresia o parálisis del nervio vago y/o nervio glosofaríngeo es una causa poco frecuente de disfunción velofaríngea.

Palabras clave: Esfínter velofaríngeo; insuficiencia velofaríngea; paladar blando; enfermedad de nervios craneales.

\begin{abstract}
Hypernasal speech and nasal regurgitation are symptoms of velopharyngeal dysfunction. This may have multiple causes, including velopharyngeal incompetence due to paresis or paralysis of the vagus nerve and/or glossopharyngeal nerve. We describe the case of a 13 year-old female patient, with hypernasal speech and acute nasal regurgitation, with a physical examination showing immobility of the right palate with no other neurological findings. Magnetic resonance imaging of the brain and lumbar puncture was normal. Transient acute velopharyngeal incompetence was diagnosed, probably of viral etiology. The patient evolved favorably with progressive clinical improvement. Velopharyngeal incompetence due to paresis or paralysis of the vagus and/or glossopharyngeal nerves is a rare cause of velopharyngeal dysfunction.
\end{abstract}

Key words: Velopharyngeal sphincter; velopharyngeal insufficiency; soft palate; cranial nerve diseases.

\footnotetext{
1 Departamento de Otorrinolaringología de la Escuela de Medicina de la Pontificia Universidad Católica de Chile.

2 Escuela de Medicina de la Pontificia Universidad Católica de Chile.
}

Los autores declaran que no existe conflicto de interés.

Recibido el 5 de agosto, 2018. Aceptado el 28 de diciembre, 2018. 


\section{INTRODUCCIÓN}

El esfínter velofaríngeo (EVF) desempeña una función fundamental para la fonación y para el proceso deglutorio. Se ubica en la zona de transición entre nasofaringe y orofaringe, siendo limitado anatómicamente por el velo del paladar, las paredes laterales de la faringe y la pared posterior de la faringe $e^{1,2}$. Durante la fonación, el velo del paladar se desplaza hacia superior y posterior, la pared faríngea posterior se mueve levemente hacia anterior y las paredes laterales de la faringe se desplazan medialmente contra el velo del paladar para así generar mayor cierre de la zona. Esto genera diferentes patrones de cierre velofaríngeo denominados circular, coronal, sagital y circular con rodete 0 anillo de Passavant ${ }^{1,3,4}$.

La motilidad del velo del paladar se produce por la acción de varios músculos, los cuales son inervados por los pares craneales $\mathrm{V}$ (trigémino), VII (facial), IX (glosofaríngeo) y X (vago)5. Los nervios craneales IX y $X$ son los que contribuyen en mayor grado para la inervación motora de los músculos velofaríngeos². La disfunción velofaríngea (DVF), es definida por Glade y cols, como "una condición donde no hay un cierre completo del EVF cuando es requerido durante la fonación" 0 existe un cierre anormalmente asimétrico, lo que permite que el aire 0 sonido se pierda 0 resuene en la cámara nasal, generando regurgitación nasal, hipernasalidad y emisión nasal de aire durante el discurso oral (voz hipernasal) $)^{3}$. Cuando hay un gap mayor 0 igual a $10 \mathrm{~mm}^{2}$ del cierre velofaríngeo se producirá este tipo de síntomas ${ }^{3,7}$. Los objetivos de este trabajo son describir un caso clínico de voz hipernasal aguda en una paciente pediátrica y su etiología, y realizar una revisión de la literatura de esta causa poco frecuente de incompetencia velofaríngea. Este trabajo cuenta con la aprobación del Comité de Ética de la Pontificia Universidad Católica de Chile.

\section{CASO CLÍNICO}

Presentamos el caso de una paciente de 13 años de edad de sexo femenino y sin antecedentes mórbidos, que consultó en nuestro servicio de otorrinolaringología por un cuadro de 5 días de evolución de voz hipernasal y regurgitación de líquidos por ambas fosas nasales, de inicio súbito. Al momento de la evaluación no refería disfonía, disfagia, disartria, hipoacusia ni vértigo. Dentro de la historia reciente, sólo cursó un cuadro de infección respiratoria alta un mes previo a la consulta. Al examen físico se apreciaba una voz hipernasal, mientras que en el examen de la orofaringe se observaba una asimetría en la elevación del velo del paladar, a expensas de una inmovilidad velopalatina a derecha (Figura 1).

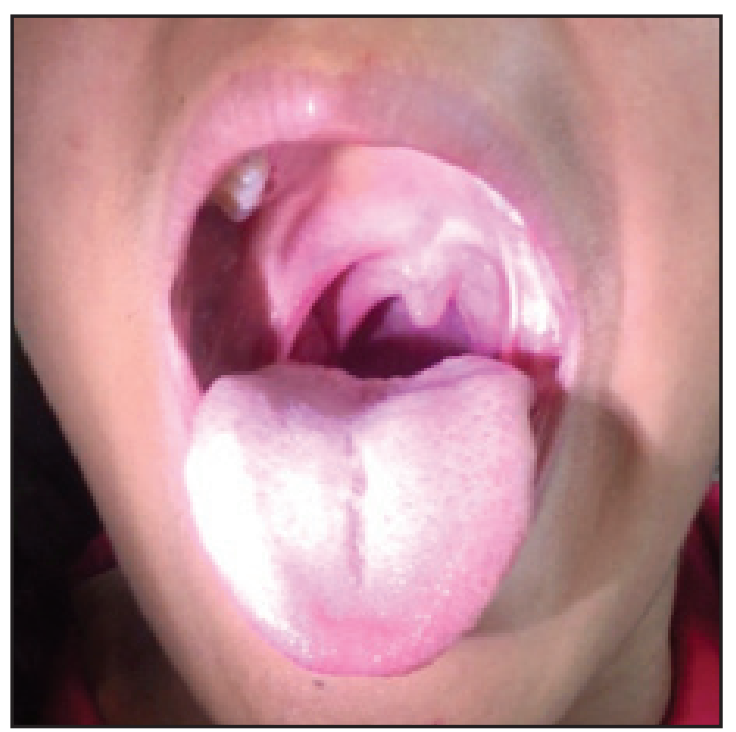

Figura 1. Evaluación del IX y X par craneano, se observa asimetría en la elevación del velo paladar al fonar, por inmovilidad a derecha. 
Además, presentaba un reflejo nauseoso disminuido y sensibilidad levemente disminuida a derecha. No se observó compromiso de otros pares craneanos ni otros hallazgos neurológicos. Se realizó una nasofibroscopía flexible en la cual se evidenció un hiato fonatorio a derecha durante la fonación de fonemas oclusivos como (K-Q) (Figura 2).

En las paredes de la rinofaringe y orofaringe se observó gran cantidad de secreciones claras retenidas, principalmente hacia la hemifaringe derecha y que no lograba limpiar con degluciones repetidas. La hipofaringe se apreciaba sin lesiones ni secreciones retenidas. A nivel laríngeo, no se observaron alteraciones anatómicas, sensitivas ni de movilidad cordal. Ante la hipótesis diagnóstica de una incompetencia velofaríngea de etiología desconocida, se decide hospitalizar para completar su estudio. Fue evaluada por el equipo de neurología infantil, con un estudio con resonancia nuclear magnética (RM) de cerebro y punción lumbar los cuales fueron normales. Se diagnosticó una incompetencia velofaríngea por mononeuropatía aguda idiopática del nervio vago y/o nervio glosofaríngeo. Se inició tratamiento con prednisona oral $40 \mathrm{mg}$ al día por 7 días, aciclovir $300 \mathrm{mg}$ cada 6 horas por 7 días y terapia fonoaudiológica. La pa- ciente evolucionó de forma favorable con mejoría clínica progresiva.

\section{DISCUSIÓN}

La DVF puede ser originada por tres mecanismos

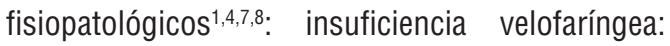
deficiencia anatómica de las estructuras del EVF. Incompetencia velofaríngea: disfunción neurofisiológica que causa una alteración de la movilidad de la velofaringe. Alteración en el aprendizaje velofaríngeo (mislearning): hipernasalidad fonemaespecífica debido a alteraciones en la articulación aprendidas, sin causas anatómicas o neurofisiológicas subyacentes. A su vez, existen múltiples etiologías para cada mecanismo (Tabla 1) 1,4,7,8.

El conocimiento de la anatomía e inervación del EVF es relevante para comprender las causas de incompetencia velofaríngea. Éste está compuesto por: el músculo tensor del velo del paladar, inervado por la división motora del nervio trigémino (V); el músculo de la úvula, inervado por los nervios palatinos menores que son ramas del nervio facial (VII) y por los músculos elevador del velo del paladar, palatofaríngeo, palatogloso y constrictor de la faringe, los cuales reciben inervación del plexo

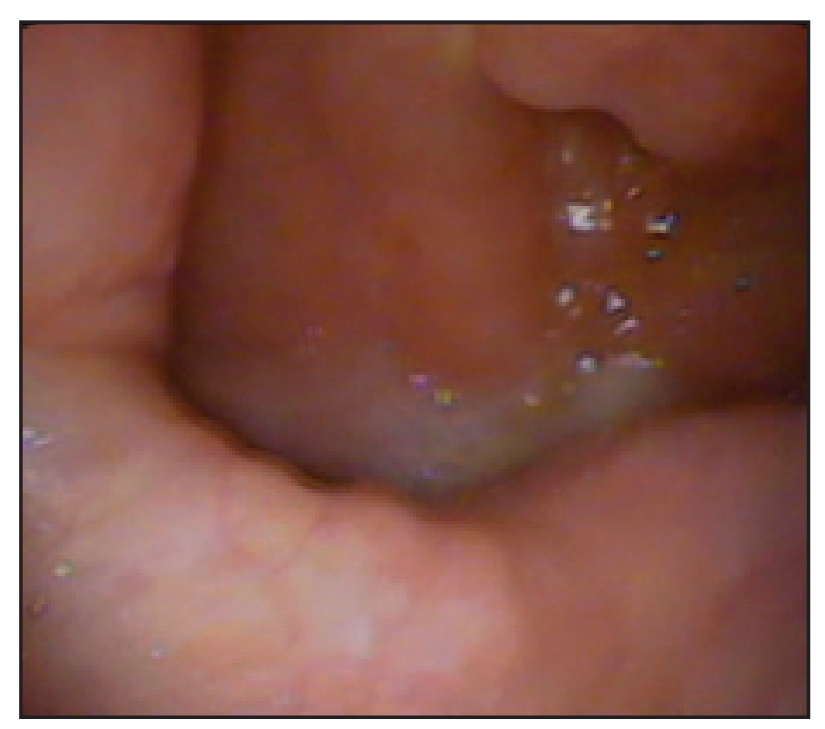

Figura 2. Nasofibroscopía por fosa nasal derecha a nivel de la rinofaringe, donde se evalúa el cierre velofaríngeo, observándose un hiato a derecha. 
Tabla 1. Causas de disfunción velofaríngea

\begin{tabular}{|lll|}
\hline Insuficiencia & Incompetencia & Alteración aprendizaje \\
\hline Fisura palatina & Hipotonía: & Articulación defectuosa \\
Fisura palatina submucosa & - Sd. velocardiofacial & Hipoacusia \\
Pared posterior faringe profunda: & - Sd. neurológicos: & \\
- Malformaciones craneofaciales & - Con disartria & \\
Atrofia adenoidea & - Con apraxia & \\
Adenoides irregulares & Paresia/parálisis pares craneanos: \\
Hipertrofia amigdalina & - AVE & \\
Posquirúrgicos: & - Proceso intracraneano \\
- Adenoidectomía & - ELA \\
- Amigdalectomía & - Enfermedad de Parkinson \\
- Avance maxilar & - Miastenia gravis & \\
- Resección tumor cavidad oral/faringe & \\
Posradiación: & \\
- Tumor cavidad oral/faringe & \\
\hline
\end{tabular}

Sd: síndrome. AVE: accidente vascular encefálico. ELA: esclerosis lateral amiotrófica.

faríngeo formado por los nervios glosofaríngeo (IX) y vago $(X)^{3,8}$. El compromiso de cualquiera de estos nervios, se manifestará con distintos grados de incompetencia velofaríngea, en especial cuando hay compromiso del IX y $\mathrm{X}$ par craneanos, que cumplen un rol principal en la movilidad del velo del paladar' ${ }^{9}$. Lesiones en el nervio glosofaríngeo resultarán en dificultad en la deglución, sentido alterado del gusto en el tercio posterior de la lengua y un reflejo faríngeo ausente en el lado afectado. Una lesión del nervio vago producirá parálisis del velo del paladar y reflejo faríngeo ausente ipsilateral ${ }^{10}$. Ambos nervios reciben proyecciones del núcleo ambiguo, núcleo motor visceral del bulbo raquídeo. Es por sus relaciones anatómicas y la semejanza en sus funciones que estos nervios se estudian en conjunto ${ }^{5}$. Villarejo y cols. exponen que, aunque es un tema aún controversial, el aporte motor del nervio IX se considera de menor importancia en comparación al nervio craneal $X$, siendo necesaria la afectación del nervio $X$ para que se observe clara debilidad velopalatina ${ }^{5,9}$. La parálisis y/o paresia unilateral de comienzo agudo y de evolución transitoria del velo del paladar es una forma infrecuente de incompetencia velofaríngea. Puede verse afectado tanto el núcleo ambiguo, como el trayecto de los nervios glosofaríngeo y/o vago y se han descrito pocos casos en la literatu$\mathrm{ra}^{2,5,10}$. El primer caso de parálisis unilateral aislada del velo del paladar fue descrito por Edin y cols. en 1976 y en la Tabla 2 se describen las principales características de reportes de casos disponibles en la literatura (Tabla 2) ${ }^{11}$.

En los casos clínicos reportados se observa el predominio en edad pediátrica, con un rango entre 2 y 18 años y que el $61 \%$ de los casos corresponden a sexo masculino. Todos presentaron como síntoma cardinal voz hipernasal y los siguientes síntomas más frecuentes fueron regurgitación nasal y disfagia. Estos datos son concordantes con la revisión realizada por Walter y cols. quienes reportaron además otros síntomas menos frecuentes como fiebre, disnea, odinofagia y cefalea ${ }^{2}$. La mayoría de los casos realizaron estudios serológicos, obteniendo sólo algunos resultados positivos para virus herpes simplex, virus coxsackie tipo $A 9$, parvovirus $B 19$, virus hepatitis $A$, enterovirus y virus Epstein-Barr. Muchos de estos virus son virus neurotrópicos lo que puede sugerir una neuropatía transitoria del nervio IX y/o X de etiología viral, sin que se sepa si la lesión se produce por una invasión directa del virus o por mecanismos inmunológicos 0 inflamatorios diferidos ${ }^{2,5}$. La inmadurez de las células neurales en la población pediátrica podría explicar la susceptibilidad de éstas a virus, apoyando la mayor incidencia en este grupo etario ${ }^{10,12,13}$. Sin embargo, el número de casos aún es muy pequeño para poder sustentar esta hipótesis y la causa aún no se ha dilucidado?

Un cuadro clínico de insuficiencia velofaríngea con las características de inicio agudo, unilateral, sin otros hallazgos neurológicos, corresponde la 
Tabla 2. Casos clínicos de incompetencia velofaríngea idiopática reportados en la literatura desde 1976 a 2018

\begin{tabular}{|c|c|c|c|c|c|c|c|c|}
\hline Autor & $\begin{array}{l}\text { Edad } \\
\text { (años) }\end{array}$ & Sexo & Síntomas & Serología & $\begin{array}{l}\text { Tiempo de } \\
\text { recuperación }\end{array}$ & Imágenes & PL & Tratamiento \\
\hline $\begin{array}{l}\text { Edin y col. } \\
(1976)^{11}\end{array}$ & $\begin{array}{l}12 \\
10 \\
7\end{array}$ & $\begin{array}{l}\text { M } \\
M \\
M\end{array}$ & $\begin{array}{l}\text { Voz hipernasal } \\
\text { Regurgitación nasal } \\
\text { Disfagia } \\
\text { Voz hipernasal } \\
\text { Regurgitación nasal } \\
\text { Disfagia } \\
\text { Voz hipernasal } \\
\text { Regurgitacion nasal }\end{array}$ & $\begin{array}{l}\mathrm{N} \\
\mathrm{N}\end{array}$ & $\begin{array}{l}2 \text { meses } \\
3 \text { semanas } \\
4 \text { semanas }\end{array}$ & $\begin{array}{l}\text { NR } \\
\text { NR }\end{array}$ & $\begin{array}{l}\mathrm{N} \\
\mathrm{NR}\end{array}$ & $\begin{array}{l}(-) \\
(-) \\
(-)\end{array}$ \\
\hline $\begin{array}{l}\text { Sullivan y col. } \\
(1976)^{16}\end{array}$ & 5 & $\mathrm{~F}$ & $\begin{array}{l}\text { Voz hipernasal } \\
\text { Disfagia }\end{array}$ & $\begin{array}{l}\text { Virus herpes } \\
\text { simplex }\end{array}$ & 3 meses & NR & N & $(-)$ \\
\hline $\begin{array}{l}\text { Nussey } \\
(1977)^{17}\end{array}$ & 12 & M & $\begin{array}{l}\text { Voz hipernasal } \\
\text { Regurgitación nasal } \\
\text { Disfagia }\end{array}$ & $\begin{array}{c}\text { Virus } \\
\text { coxsackie A9 }\end{array}$ & 1 semana & NR & NR & NR \\
\hline $\begin{array}{l}\text { Roberton y } \\
\text { col. }(1982)^{18}\end{array}$ & $\begin{array}{l}9 \\
6\end{array}$ & $\begin{array}{l}\text { M } \\
M\end{array}$ & $\begin{array}{l}\text { Voz hipernasal } \\
\text { Regurgitación nasal } \\
\text { Disfagia } \\
\text { Voz hipernasal } \\
\text { Regurgitación nasal } \\
\text { Disfagia }\end{array}$ & $\mathrm{N}$ & $\begin{array}{l}2 \text { semanas } \\
6 \text { semanas }\end{array}$ & $\begin{array}{l}\text { NR } \\
\text { NR }\end{array}$ & $\begin{array}{l}\text { NR } \\
\text { NR }\end{array}$ & $\begin{array}{l}(-) \\
(-)\end{array}$ \\
\hline $\begin{array}{l}\text { Crovetto y. } \\
\text { col }(1987)^{19}\end{array}$ & 12 & M & $\begin{array}{l}\text { Voz hipernasal } \\
\text { Regurgitacion nasal }\end{array}$ & N & 3 semanas & TAC & NR & Prednisolona \\
\hline $\begin{array}{l}\text { Cuvellier y } \\
\text { col. }(1998)^{12}\end{array}$ & $\begin{array}{l}2 \\
6 \\
6\end{array}$ & $\begin{array}{l}\mathrm{F} \\
\mathrm{F} \\
\mathrm{M}\end{array}$ & $\begin{array}{l}\text { Voz hipernasal } \\
\text { Regurgitacion nasal } \\
\text { Voz hipernasal } \\
\text { Voz hipernasal } \\
\text { Regurgitacion nasal }\end{array}$ & $\begin{array}{l}\mathrm{N} \\
\mathrm{NR} \\
\mathrm{NR}\end{array}$ & $\begin{array}{l}3 \text { semanas } \\
4 \text { semanas } \\
3 \text { semanas }\end{array}$ & $\begin{array}{l}\text { RNM } \\
\text { RNM } \\
\text { TAC }\end{array}$ & $\begin{array}{c}N \\
N \\
N R\end{array}$ & $\begin{array}{c}(-) \\
(-) \\
\text { Prednisona }\end{array}$ \\
\hline $\begin{array}{l}\text { Mon y col. } \\
(2000)^{20}\end{array}$ & 18 & M & $\begin{array}{l}\text { Voz hipernasal } \\
\text { Disfagia }\end{array}$ & N & 3 semanas & RNM & $\begin{array}{l}\lg G \% \\
\text { e índice } \\
\text { IgG- } \\
\text { albúmina } \\
\text { elevado }\end{array}$ & $\begin{array}{l}\text { Glicerol } \\
\text { y } \\
\text { esteroides }\end{array}$ \\
\hline $\begin{array}{l}\text { Villarejo y } \\
\text { col. }(2002)^{5}\end{array}$ & 12 & $F$ & $\begin{array}{l}\text { Voz hipernasal } \\
\text { Regurgitación nasal } \\
\text { Disfagia }\end{array}$ & N & 3 semanas & RNM & $(-)$ & $(-)$ \\
\hline $\begin{array}{l}\text { Auvin y col. } \\
(2003)^{15}\end{array}$ & $\begin{array}{l}\text { a. } 5 \\
\text { b. } 6\end{array}$ & M & $\begin{array}{l}\text { a.Voz hipernasal } \\
\text { Regurgitación nasal } \\
\text { Disfagia } \\
\text { b.Voz hipernasal } \\
\text { Regurgitación nasal } \\
\text { Disfagia }\end{array}$ & $\begin{array}{l}\text { a.NR } \\
\text { b.(-) }\end{array}$ & $\begin{array}{c}\text { a. } 3 \text { semanas } \\
\text { b. NR }\end{array}$ & $\begin{array}{l}\text { a.NR } \\
\text { b.NR }\end{array}$ & $\begin{array}{l}\text { a.NR } \\
\text { b.NR }\end{array}$ & $\begin{array}{c}\text { a. }(-) \\
\text { b. } \\
\text { Prednisolona }\end{array}$ \\
\hline $\begin{array}{l}\text { González y } \\
\text { col. }(2003)^{21}\end{array}$ & 5 & $\mathrm{~F}$ & $\begin{array}{l}\text { Voz hipernasal } \\
\text { Regurgitación nasal } \\
\text { Disfagia }\end{array}$ & N & 10 días & RNM & NR & $(-)$ \\
\hline
\end{tabular}




\begin{tabular}{|c|c|c|c|c|c|c|c|c|}
\hline Autor & $\begin{array}{l}\text { Edad } \\
\text { (años) }\end{array}$ & Sexo & Síntomas & Serología & $\begin{array}{l}\text { Tiempo de } \\
\text { recuperación }\end{array}$ & Imágenes & $P L$ & Tratamiento \\
\hline $\begin{array}{l}\text { Jary y col. } \\
(2004)^{22}\end{array}$ & $\begin{array}{l}10 \\
11\end{array}$ & $\begin{array}{l}F \\
F\end{array}$ & $\begin{array}{l}\text { Voz hipernasal Disfagia } \\
\text { Voz hipernasal Regurgitación nasal }\end{array}$ & $\begin{array}{l}\mathrm{N} \\
\mathrm{N}\end{array}$ & $\begin{array}{l}12 \text { meses } \\
6 \text { meses }\end{array}$ & $\begin{array}{l}\text { RNM } \\
\text { NR }\end{array}$ & $\begin{array}{l}\text { NR } \\
\text { NR }\end{array}$ & $\begin{array}{l}(-) \\
(-)\end{array}$ \\
\hline $\begin{array}{l}\text { Alp y col. } \\
(2005)^{23}\end{array}$ & 5 & $F$ & $\begin{array}{l}\text { Voz hipernasal } \\
\text { Regurgitación nasal } \\
\text { Disfagia }\end{array}$ & N & 45 días & RNM & N & $(-)$ \\
\hline $\begin{array}{l}\text { Soares y col. } \\
(2006)^{24}\end{array}$ & 8 & M & $\begin{array}{l}\text { Voz hipernasal } \\
\text { Regurgitación nasal } \\
\text { Disfagia }\end{array}$ & $\begin{array}{l}\text { Parvovirus } \\
\text { B19 }\end{array}$ & 10 semanas & RNM & N & $(-)$ \\
\hline $\begin{array}{l}\text { Fleta y col. } \\
(2006)^{25}\end{array}$ & $\begin{array}{l}12 \\
7\end{array}$ & $\begin{array}{l}\mathrm{F} \\
\mathrm{M}\end{array}$ & $\begin{array}{l}\text { Voz hipernasal } \\
\text { Regurgitación nasal } \\
\text { Disfagia } \\
\text { Voz hipernasal } \\
\text { Regurgitación nasal } \\
\text { Disfagia }\end{array}$ & $\begin{array}{l}\mathrm{N} \\
\mathrm{N}\end{array}$ & 5 días & RNM & $\begin{array}{l}\text { NR } \\
\text { NR }\end{array}$ & $\begin{array}{l}\text { NR } \\
(-)\end{array}$ \\
\hline $\begin{array}{l}\text { Prasad y col. } \\
(2007)^{26}\end{array}$ & 8 & M & Voz hipernasal & $\begin{array}{c}\text { Virus } \\
\text { hepatitis A }\end{array}$ & 4 semanas & RNM & NR & $(-)$ \\
\hline $\begin{array}{l}\text { Thapa y col. } \\
(2009)^{27}\end{array}$ & 5 & $F$ & Voz hipernasal & $\begin{array}{c}\text { Virus } \\
\text { hepatitis A }\end{array}$ & 7 días & RNM & N & $(-)$ \\
\hline $\begin{array}{l}\text { Sondhi y col. } \\
(2011)^{28}\end{array}$ & 10 & M & $\begin{array}{l}\text { Voz hipernasal } \\
\text { Regurgitación nasal } \\
\text { Disfagia }\end{array}$ & NR & 11 días & RNM & N & Prednisolona \\
\hline $\begin{array}{l}\text { Yoshihara y co } \\
(2012)^{29}\end{array}$ & & M & $\begin{array}{l}\text { Voz hipernasal } \\
\text { Regurgitacion nasal }\end{array}$ & N & 15 semanas & RNM & N & Prednisolona \\
\hline $\begin{array}{l}\text { Walter y col. } \\
(2013)^{2}\end{array}$ & 9 & $\mathrm{~F}$ & Voz hipernasal & $\begin{array}{c}\text { Borrelia } \\
\text { burgdorferi: } \\
\text { leve elevación } \\
\text { IgG, IgM } \\
\text { normal }\end{array}$ & 3 semanas & RNM & NR & Doxiciclina \\
\hline $\begin{array}{l}\text { Ruchi Verma } \\
\text { y col. } \\
(2015)^{30}\end{array}$ & 12 & M & $\begin{array}{l}\text { Voz hipernasal } \\
\text { Regurgitación nasal } \\
\text { Disfagia }\end{array}$ & NR & 10 días & NR & NR & Prednisolona \\
\hline $\begin{array}{l}\text { Singh y col. } \\
(2015)^{13}\end{array}$ & 15 & M & $\begin{array}{l}\text { Voz hipernasal } \\
\text { Regurgitación nasal }\end{array}$ & N & 9 días & RNM & N & Prednisolona \\
\hline $\begin{array}{l}\text { Gupta y col. } \\
(2015)^{31}\end{array}$ & 17 & $\mathrm{~F}$ & $\begin{array}{l}\text { Voz hipernasal } \\
\text { Regurgitación nasal }\end{array}$ & $\begin{array}{c}\text { Virus } \\
\text { Epstein-Barr }\end{array}$ & $1 \mathrm{mes}$ & RNM & NR & $(-)$ \\
\hline $\begin{array}{l}\text { Brecheteau } \\
(2015)^{32}\end{array}$ & 12 & M & $\begin{array}{l}\text { Voz hipernasal } \\
\text { Regurgitacion nasal }\end{array}$ & $N$ & $1 \mathrm{mes}$ & RNM & $\begin{array}{c}\text { Entero } \\
\text { virus }\end{array}$ & Prednisona \\
\hline $\begin{array}{l}\text { Ahmed y col. } \\
(2017)^{10}\end{array}$ & 10 & M & $\begin{array}{l}\text { Voz hipernasal } \\
\text { Regurgitación nasal }\end{array}$ & N & 10 días & RNM & NR & Prednisolona \\
\hline $\begin{array}{l}\text { Güngör y col. } \\
(2017)^{14}\end{array}$ & 17 & M & $\begin{array}{l}\text { Voz hipernasal } \\
\text { Regurgitacion nasal }\end{array}$ & N & 1 mes & RNM & NR & $(-)$ \\
\hline $\begin{array}{l}\text { Thone y col. } \\
(2018)\end{array}$ & 13 & $\mathrm{~F}$ & $\begin{array}{l}\text { Voz hipernasal } \\
\text { Regurgitación nasal }\end{array}$ & $(-)$ & 2 meses & RNM & N & $\begin{array}{l}\text { Prednisona } \\
\text { Aciclovir }\end{array}$ \\
\hline
\end{tabular}

NR =no reportado; $N$ =normal; RNM: resonancia nuclear magnética; PL =punción lumbar; (-) =sin tratamiento. 
mayoría de las veces a un proceso autolimitado, benigno y de causa viral o idiopática ${ }^{5}$. Sin embargo, frente a un diagnóstico etiológico incierto es relevante descartar procesos progresivos como lesiones expansivas de tronco cerebral 0 ángulo pontocerebeloso (neoplásicas o infecciosas), lesiones isquémicas, enfermedades desmielinizantes, enfermedades neuromusculares u otros síndromes infantiles como la enfermedad de Duchenne 0 el síndrome de Moebius 2,10. Para descartar estas etiologías, el estudio en nuestro caso incluyó el uso de RNM y punción lumbar. En la Tabla 2 podemos observar que en la gran mayoría de los casos se solicitó también un estudio imagenológico y en un tercio de ellos se realizó punción lumbar donde sólo un caso salió positivo para enterovirus (Tabla 2).

Con respecto al tratamiento, aproximadamente la mitad de los casos publicados no recibió ningún tipo de terapia, a pesar de lo cual se logró una recuperación completa de los síntomas. La otra mitad en cambio, recibió tratamiento, la mayoría con corticoides y hubo un caso tratado con glicerol y un caso con antibiótico, este último por una leve elevación de IgG para Borrelia burgdorferi. Nuestro caso fue tratado con corticoides y el único que fue tratado además con aciclovir (Tabla 2). La revisión de Walter y cols. tiene resultados muy similares². El pronóstico de esta patología es en general benigno. Nuestra revisión reportó recuperación en todos los casos, pero con una amplia variación con respecto al tiempo de recuperación, el que se va

\section{BIBLIOGRAFÍA}

1. Kummer AW. Types and causes of velopharyngeal dysfunction. Semin Speech Lang 2011; 32: 150-8.

2. Walter V, Nisa L, Leuchter I. Acute isolated velopharyngeal insufficiency in children: Case report and systematic review of the literature. Eur Arch Oto-Rhino-Laryngology 2013; 270: 1975-80.

3. Glade RS, Deal R. Diagnosis and Management of Velopharyngeal Dysfunction. Oral Maxillofac Surg Clin North Am 2016; 28: 181-8.

4. Prada JR, García T, Echeverri MP, tavera MC. Patrones de cierre velofaringeo: Estudio comparativo entre población sana y pacientes con paladar hendido. Cir Plast Ibero-Latinoamericana 2010; 36: 305-12. entre 5 días y 12 meses. Walter y cols reportaron que $65 \%$ de los casos presentó recuperación completa y $35 \%$ con recuperación parcial, seguimiento que se realizó en un periodo entre 1 semana hasta 2,5 años $^{2,14}$. En el caso de nuestra paciente, ella tuvo una mejoría total a los 2 meses (Tabla 2). Se describe sólo un caso clínico de Auvin y cols. donde el paciente tuvo recurrencia del cuadro 11 meses después del primer episodio, no obstante, en ambas ocasiones obtuvo recuperación completa ${ }^{15}$.

\section{CONCLUSIÓN}

Es relevante distinguir y reconocer los diferentes tipos de disfunción velofaríngea y sus causas, ya que el tratamiento y pronóstico de las distintas etiologías son diferentes. La parálisis o paresia aguda, unilateral y aislada del velo del paladar es una causa poco frecuente de voz hipernasal y regurgitación en niños y se ha descrito como una condición benigna y autolimitada en la mayoría de los casos. Las causas no están claramente establecidas, aunque etiologías virales han sido sugeridas. El diagnóstico debe realizarse luego de un estudio completo del paciente, habiéndose descartado otras causas más graves. Además, es de suma importancia el seguimiento y monitorización de la función velofaríngea del paciente para diagnosticar precozmente patologías tumorales 0 progresión de síntomas.

5. Villarejo-Galende A, Camacho-Salas A, PenasPrado M et AL. Parálisis aislada unilateral del velo del paladar: presentación de un caso y revisión de la literatura. Rev Neurol 2003; 36: 337-9.

6. Ruda JM, Krakovitz P, Rose AS. A Review of the Evaluation and Management of Velopharyngeal Insufficiency in Children. Otolaryngol Clin North Am 2012; 45: 653-69.

7. Alvo A, Sedano C. Complicaciones velofaríngeas de adenoamigdalectomía. Rev Otorrinolaringol y Cirugía Cabeza y Cuello 2015; 75: 286-94.

8. Sih T, Sakano E, Hayashi L, Morelló G. Insuficiencia y disfunción velofaríngea. En: Morelló-Castro G. Otorrinolaringología Pediátrica. Pringer Verlag Ibérica; 1999. p. 423-9.

9. Snell R. Los núcleos de los nervios craneales, 
sus conexiones centrales y distribución. En: Snell R. Neuroanatomía clínica. Buenos Aires: Editorial Médica Panamericana S.A.; 2007. p. 357-401.

10. Ahmed S, Hussain A, Patel T, Ahmed HR, Shariff MI, KafIL MY. Idiopathic palatal palsy. J Family Med Prim Care 2017; 6: 437-8.

11. Edin M, Sveger T, Tegner H, Tuernström 0. Isolated temporary pharyngeal paralysis in childhood. Lancet 1976; 1: 1047-49.

12. Cuvellier JC, Cuisset JM, Nuyts JP, Vallee L. Acquired and isolated asymmetrical palatal palsy. Neuropediatrics 1998; 29: 324-5.

13. Singh H, Mathur R, Kaur P. Isolated palatal palsy: a clinical rarity. Neuroimmunol Neuroinflammation 2015; 2: 190-2.

14. Güngör 0 , Kirik $S$, IsikaY S, Güngör G, Bilal N. Idiopathic Unilateral Paralysis of the Palate in a Child: a Case Report. Pediatr Emerg Care 2017; 6: 1.

15. Auvin S, Cuvellier JC, ValléE L. Isolated recurrent palatal palsy in a child. Neuropediatrics 2003; 34 : 278-9.

16. Sullivan JL, Carlson CB. Isolated temporary pharyngeal paralysis in childhood. Lancet 1976; 2: 863.

17. NusSEY AM. Paralysis of palate in a child. Br Med J 1977; 2: 165-6.

18. Roberton DM, MeLlor DH. Asimmetrical palatal paresis in childhood: A transient cranial mononeuropathy? Develop Med Child Neurol 1982; 24: 842-9.

19. Crovetto MA, Aguirre JM, Municio A, Pérez-Rojo A, SAInT-Gerons $S$. Idiopathic paralysis of the palate in chidhood. Br J Oral Maxillofac Surg 1988; 26 : 241-3.

20. Mon Y, Nakamura N. Velopharyngeal palsy: a case report. Rinsho Shinkeigaku 2000; 40: 364-6.

21. González Alvarez V, Costa Orvay Ja, Guardia Camí MT, Garrido Romero R, Pineda Marfá M, Luaces Cubells C. Idiopathic palatopharyngeal hemiparalysis. An Pediatr (Barc) 2003; 59: 595-8.
22. Jary A, Maillard L, Raffo E, Ducroco X, Braun $M$, Vespignani H. Acute, isolated and reversible paralysis of the soft palate in childhood. Rev Neurol (Paris) 2004; 160: 836-8.

23. Alp $H$, Tan H, Altunkaynak $S$, Orbak Z. Idiopathic unilateral paralysis of the palate in childhood. Pediatr Neurol 2005; 33: 134-5.

24. Soares-Fernandes JP, Maré R. Isolated velopalatine paralysis associated with parvovirus B19 infection. Arq Neuropsiquiatr 2006; 64: 603-5.

25. Fleta Zaragozano J, Jiménez Vidal A, Meavilla Olivares S, Alonso Curcó X, Olivares lópez JL, Alfonso Collado I. Idiopathic soft palate hemiparalysis. Ann Pediatr 2006; 65: 623-5.

26. Prasad PL, Prasad AN, Patnalk SK. Unilateral palatal palsy with viral hepatitis. Indian J Pediatr 2007; 74: 1039-40.

27. Thapa R, Biswas B, Ghosh A, et al. Unilateral palatal and abducens palsy in childhood hepatitis A virus infection. J Child Neurol 2009; 24: 628-9.

28. SondHI V, PAtNAIK SK. Isolated idiopathic unilateral paralysis of soft palate and pharynx. Indian Pediatr. 2011; 48: 237-9.

29. Yoshihara N, Okuda M, Takano K, Wada T, Osaka $\mathrm{H}$. Idiopathic cranial polyneuropathy with unilateral $I X$ and $X$ and contralateral $X I$ nerve palsy in a 4-year-old boy. Pediatr Neurol 2012; 47: 198200.

30. Ruchi Verma, Vidya H. Isolated Idiopathic Unilateral Paralysis of Soft Palate: A Case Report. Journal of Evolution of Medical and Dental Sciences 2015; 4: 5234-6.

31. Gupta R, Gupta R, Sethi S, Khanal M. Isolated Unilateral Soft Palate Palsy following Tonsillopharyngitis Caused by Epstein-Barr Virus Infection. The Cleft Palate-Craniofacial Journal 2017; 54: 351-3.

32. Brecheteau C, Laccourreye L, Gueden S, Breheret R. Paralysie vélaire aiguë réversible de l'enfant : à propos d'un cas. Arch Pediatr 2015; 22: 544-6. 